\title{
Measures of postural stability
}

\author{
Hans Chaudhry, PhD; Thomas Findley, MD, PhD; Karen S. Quigley, PhD; Bruce Bukiet, PhD; Zhiming Ji, PhD; \\ Tiffany Sims, MS; Miriam Maney, MS \\ War-Related Illness and Injury Study Center, Department of Veterans Affairs Medical Center, East Orange, NJ; \\ Departments of Biomedical Engineering and Mechanical Engineering, New Jersey Institute of Technology, Newark, NJ; \\ Department of Psychiatry, University of Medicine and Dentistry of New Jersey-New Jersey Medical School, Newark, \\ NJ; Department of Mathematical Sciences and Center of Applied Mathematics and Statistics, New Jersey Institute \\ of Technology, Newark, $N J$
}

\begin{abstract}
Dynamic posturography has become an important tool for understanding standing balance in clinical settings. A key test in the NeuroCom International (Clackamas, Oregon) dynamic posturography system, the Sensory Organization Test (SOT), provides information about the integration of multiple components of balance. The SOT test leads to an outcome measure called the "equilibrium score" (ES), which reflects the overall coordination of the visual, proprioceptive, and vestibular systems for maintaining standing posture. Researchers, therapists, and physicians often use the ES from the SOT as a clinically relevant measure of standing balance. We discuss here the formula used for evaluating the ES and propose an additional measure of postural stability, called the Postural Stability Index (PSI), that accounts for shear force and individual anthropomorphic measures. We propose that this new measure provides a clinically important adjunct to the current SOT and can be calculated from data already collected by the NeuroCom forceplate during the SOT.
\end{abstract}

Key words: dynamic posturography, equilibrium, mathematical model, medically unexplained symptoms, posture, standing balance, veterans.

\section{INTRODUCTION}

Postural control under static conditions is usually called "postural steadiness," whereas the dynamic postural response to applied or volitional perturbations is called "postural stability" [1]. Dynamic posturography $[2,3]$ has become an important tool for understanding standing balance in clinical settings. A key test in the NeuroCom International (Clackamas, Oregon) dynamic posturography system, the Sensory Organization Test (SOT), provides information about the integration of the visual, proprioceptive, and vestibular components of balance, which leads to an outcome measure called the "equilibrium score" (ES), reflecting the overall coordination of these systems to maintain standing posture. Postural stability is an essential component in assessing the efficacy of interventions for improving balance [4,5].

\footnotetext{
Abbreviations: $\mathrm{CFS}=$ chronic fatigue syndrome, $\mathrm{COM}=$ center of mass, COP = center of pressure, ES = equilibrium score, IRB = institutional review board, PSI = Postural Stability Index, SOT = Sensory Organization Test.
}

This material was based on work supported by a pilot grant to K. Quigley under National Institutes of Health parent grant U01AI 32247 to B. Natelson.

Address all correspondence to Karen S. Quigley, PhD; Department of Psychiatry, UMDNJ-New Jersey Medical School, Newark, NJ 07107; 973-676-1000, ext. 1-2719; fax: 973-3957114; email: quigleks@umdnj.edu.

DOI: 10.1682/JRRD.2003.09.0140 
Currently, physicians, therapists, and researchers often use the ES from the SOT to assess the postural stability of a patient or subject. Because the SOT-based ES does not consider some key biomechanical aspects of postural stability, we propose a new measure of postural stability, which we call the "Postural Stability Index" (PSI). We first provide a brief description of the NeuroCom EquiTest dynamic posturography system and the ES derived from this system. Then we propose and describe a new index of postural stability that accounts for additional biomechanical properties of standing that should be reflected in a clinically meaningful score.

The NeuroCom EquiTest system consists of a support surface and a visual surround. The EquiTest device performs an SOT with six conditions: conditions 1, 2, and 3 with the platform fixed and conditions 4, 5, and 6 with the platform moving. When the platform moves, it is referenced to the subject's sway, such that as the individual leans forward, the platform tilts forward to minimize the degree of changed proprioceptive input from the selfgenerated sway. This platform adjustment is called "sway-referenced motion." Similarly, in conditions where the visual surround moves, the surround is referenced to the person's sway to minimize the ability to obtain visually relevant information about how far the individual is from the vertical. In other conditions, the visual input is removed instead by asking the subject to close his or her eyes. Participants are asked to stand quietly and steadily for three trials each of the following six conditions: (1) eyes open, surround and platform stable; (2) eyes closed, surround and platform stable; (3) eyes open, sway-referenced surround; (4) eyes open, swayreferenced platform; (5) eyes closed, sway-referenced platform; and (6) eyes open, sway-referenced surround and platform.

The NeuroCom Equitest system calculates ES for each trial in each condition according to equation (1):

$$
\mathrm{ES}=\left\{12.5-\left[\theta_{\max }(\text { ant })-\theta_{\max }(\text { post })\right]\right\} / 12.5,
$$

where $\theta_{\max }$ (ant) is the maximum anterior sway angle in degrees during a trial, $\theta_{\max }$ (post) is the maximum posterior sway angle in degrees during the same trial, 12.5 is the limit of sway in degrees in the sagittal plane for normal stance, and $12.5^{\circ}$ is assumed to be the limit of stability for a normal individual (found in section PO-5 of the appendix portion of the SmartEquitest system operator's manual
[6]). However, this angle may vary depending upon the age, sex, mass, and height of the individual. We also know, from experimental results (found in section PO-3 of the appendix portion of the Balance Master operator's manual [7]), that functional stability limits for the average adult subject are approximately $7^{\circ}$ anteriorly and $5^{\circ}$ posteriorly. Thus, an asymmetry in the usual limit of stability exists that is disregarded in the ES calculation.

No movement of the subject results in a perfect score of 100. If the subject falls or the value of the ES is negative, the subject receives a score of 0 . Thus, the ES ranges between 0 and 100. However, for some subjects, the limit of sway may be more than $12.5^{\circ}$ - say, $14^{\circ}$ and in that case the ES will be negative (although in practice, the ES is given a value of 0 ). Thus, the assumptions about the overall magnitude of the limits of stability as well as the magnitude of anterior and posterior sway can introduce errors into the ES calculation for individuals whose limits of stability vary significantly from the ageand height-matched norms.

The Composite ES is evaluated as a weighted average of the scores from the six conditions of the SOT of a subject, where each condition consists of three identical 20 s trials with force data sampled at $100 \mathrm{~Hz}$. Because the Composite ES is composed of information from both static conditions ( 1 and 2 ) and dynamic conditions (3, 4, 5 , and 6), this composite index may obscure important information about independent aspects of static and dynamic balance. In addition, many combinations of anterior and posterior sway across the same overall range can give the same ES. For example, the overall limit of stability of $6^{\circ}$ can be made up of the many combinations (e.g., $6^{\circ}$ of anterior sway and $0^{\circ}$ of posterior sway, or $3^{\circ}$ of anterior sway and $-3^{\circ}$ of posterior sway, etc.). These combinations would result in the same Composite ES. Yet, a subject with a $+6 / 0^{\circ}$ of sway combination likely has a greater risk of falling than a subject with a $+3 /-3$ combination, since the former is close to the functional stability limit on the anterior side, whereas the second combination is not close to the functional stability limit on either side. Therefore, the ES, which would be identical in these two situations, can be insensitive to functionally relevant differences in postural stability. For an assessment of postural stability, the formula for a stability measure should include important biomechanical information, such as mass and height of the subject and ankle torque produced to maintain stability. These are absent in the formula for ES. Our study devises a formula to reflect 
postural stability that incorporates a broader range of biomechanical aspects of upright stance, and does not have the ambiguities of the ES.

Researchers have used measures other than Composite ES for assessing postural stability. A stability measure for quiet standing in able-bodied subjects was proposed by Popovic et al., who used measures of the center of pressure (COP) to find four stability zones: high preference, low preference, undesirable, and unstable [8]. The boundaries of stability zones were modeled with the use of ellipses to capture the two-dimensional form and orientation of the stability zone. However, in practice, physicians find it difficult to identify these stability zones to assess postural stability of a patient. Alexander and colleagues suggested a single measure for postural stability by measuring the rate at which consecutive peak values of the total angular momentum of all body segments about the ankles diminish when a standing person is subjected to various types of perturbations [9]. Shepard and colleagues used this method to compare the instability of young and elderly adults [10]. However, this method does not provide a defined measure of stability or instability.

We believe that, in a clinical setting, a single number or a small set of numbers representing postural stability is desirable so that clinicians can quickly determine whether a patient requires a balance intervention or whether an intervention has been effective in improving postural stability. Our study proposes a single measure defining postural stability that is based on the physics of standing and that makes fewer assumptions than ES.

\section{METHODS}

To assess postural stability, we must consider the effort needed to maintain stability across an entire test of dynamic balance where the platform or visual environment is altered to perturb balance. We must consider the total value of the stabilizing torque to counteract the destabilizing torque due to gravity in quiet standing. Therefore, we propose and evaluate a new measure of postural stability, the PSI. We define the PSI as the percentage ratio of the total stabilizing ankle torque and the total destabilizing torque due to gravity (obtained from the product of the weight, height, and the sway angle) during quiet standing in any of the six conditions. A value of 100 indicates perfect stability. The amount of instability is reflected in how much the PSI is less than 100, and the range of PSI is 0 to100. In mathematical terms, we have

$$
\text { PSI }=\Sigma|M g h \theta| / \Sigma|\tau|
$$

where $M$ is the mass of the subject, $g$ is acceleration due to gravity, $h$ is 0.55 times the height of the subject (the average distance of center of mass [COM] from the platform, based on anthropometric data), $\tau$ is the stabilizing torque at the ankle, the vertical bars indicate the absolute value, $\Sigma$ is the summation of the values inside the bars, and $\theta$ is the sway angle in radians at any time $t$ during the test (see our two-link model [11]). In Equation (2), when the numerator and denominator are equal, the PSI is 100 and the subject is perfectly stable. One can use Equation (2) to independently calculate a PSI value for each condition.

The parameters involved in Equation (2) can be seen in Figures 1 and 2. Here, $F_{F}$ and $F_{R}$ are the front and rear vertical ground reaction forces, $F_{H}$ is the shear force, and $d$ and $e$ are as shown in Figure 2. $a$ is the perpendicular distance from the line through the ankle and pin joints to the center of mass of the foot (not shown in Figure 2 because it is quite small).

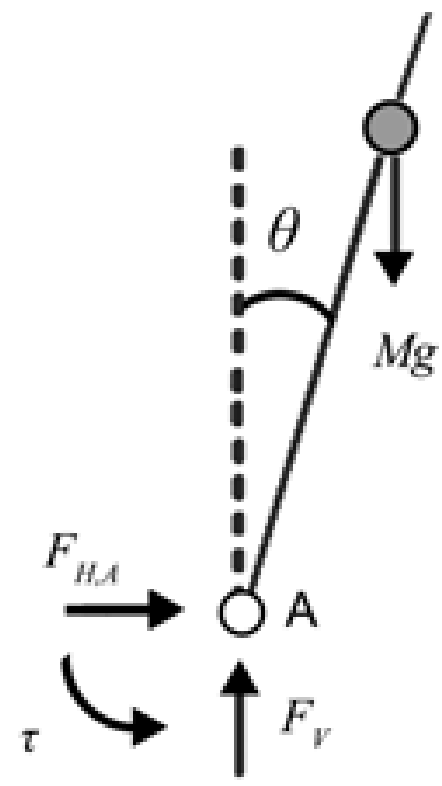

Figure 1.

Free body diagram of body (above ankle). Ankle is at small open circle. $\mathrm{A}=$ ankle, $\theta=$ sway angle, $M=$ mass of body above ankle joint, $g=$ acceleration due to gravity, $F_{H, A}=$ horizontal force acting at the ankle joint, $\tau=$ stabilizing torque at ankle, and $F_{V}=$ vertical force at ankle joint. 


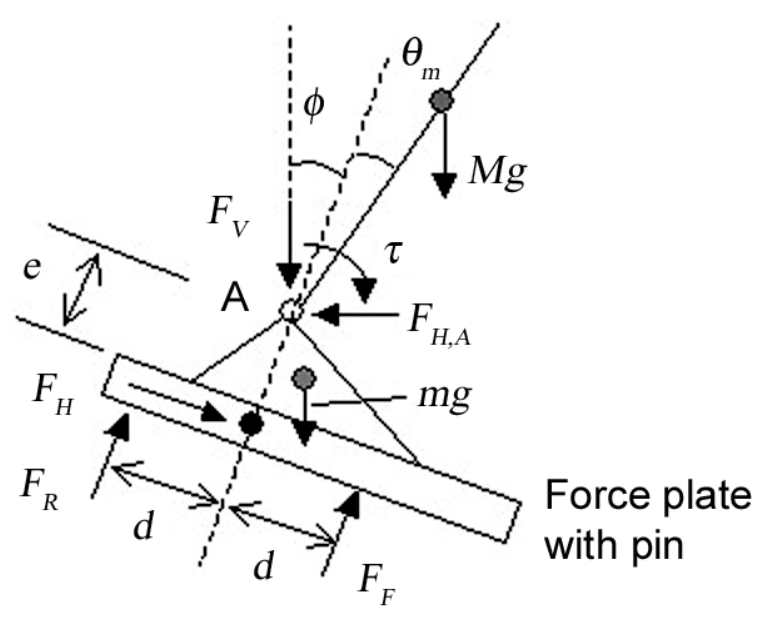

Figure 2.

Free body diagram of feet with force plate. $d$ = distance from force transducer to pin axis on force plate; $\phi=$ rotation angle of force plate during sway referenced motion; $\theta_{m}=$ measured relative sway angle with respect to line perpendicular to force plate; $F_{V}=$ vertical force at ankle joint; $M$ = mass of body above ankle joint; $g$ = acceleration due to gravity; $\tau=$ stabilizing torque at ankle; $e=$ distance from the horizontal force transducer to the ankle joint; $\mathrm{A}=$ ankle; $F_{H, A}=$ horizontal force acting at the ankle joint; $F_{H}=$ horizontal reaction force (shear force) measured with force transducer at pin joint of force plate; $m=$ total mass of feet and force plate; and $F_{R}$ and $F_{F}=$ ground reaction forces perpendicular to the force plate, measured with front and rear transducers, respectively.

Recall that two individuals with different magnitudes of anterior and posterior sway but with the same overall sway range will have the same ES [Equation (1)] but a different PSI [Equation (2)]. That is because ES depends only on the overall sway range, whereas PSI depends on the entire sway history and on the individual's mass, height, and torque at the ankle. Thus, the PSI relies on biomechanical data recorded from each individual, whereas the ES relies heavily on normative assumptions.

\section{RESULTS}

For the sake of an example, we take the data of a normal healthy subject (one of the authors of this manuscript) in a trial $20 \mathrm{~s}$ long for each of the six conditions of the SOT, for which we use our previously developed two-link model [11]). The values of the anthropometric parameters for this subject are $M=73.325 \mathrm{~kg}, m=2.265 \mathrm{~kg}, I=85.02 \mathrm{~kg}(\mathrm{~m})^{2}$, $h=0.933 \mathrm{~m}, d=0.107 \mathrm{~m}, e=0.065 \mathrm{~m}$, and $a=0.0315 \mathrm{~m}$.
The sampling frequency is $100 \mathrm{~Hz}$. For this model, the sway angle and torque at 2,000 data points are given by

$$
\theta=\frac{M h\left[\left(F_{F}-F_{R}\right) d+F_{H} e-m g a\right]+I \cdot F_{H}}{M^{2} g h^{2}-I\left[(M+m) g-\frac{F_{F}+F_{R}}{k+1}\right]},
$$

and

$$
\tau=\left(F_{F}+F_{R}\right) d+F_{H} e-m g a \cos \frac{k \theta}{k+1} .
$$

In Equations (3) and (4), $I$ is the moment of inertia about the ankle joint, $k$ is the gain factor where $k=0$ for test conditions 1,2 , and 3 (i.e., when the platform is fixed), and $k=1$ for conditions 4,5 , and 6 (i.e., when the platform is moving); and the last term in the denominator, i.e., $\left(F_{F}+F_{R}\right) / k+1$ [Equation (3)] must be divided by 2 for a moving platform.

The use of a two-link model for torque and sway angle instead of a single-link model is justified because the torque is not evaluated in the Equitest system. The Equitest system uses a single-link model, in which the foot is taken as a fixed point (ankle joint and heel coincide) and the body sways about this fixed point like an inverted pendulum. In this simplified pendulum model, the stabilizing torque $(\tau)$ equals the destabilizing torque $(M g h \theta)$, which one can easily verify from Equations (3) and (4) of this paper by taking $a=e=0$ (since the ankle joint and heel are considered a single point in a single-link model); $k=0$ (for fixed platform); $F_{H}=0$ (since horizontal force is ignored in the NeuroCom single-link model); and $(M+m) g=F_{F}+F_{R}$. Thus our concept of PSI as the ratio between stabilizing and destabilizing torque will always be equal to 1 in the single-link model. This is not the situation in our two-link model. With the use of the two-link model, the ankle joint and heel are separate points and the body sways about the ankle joint as it occurs physiologically.

The value of $\theta$ in the Equitest system is not correctly evaluated for the following reasons, noted in our earlier paper [11]:

1. Horizontal forces do not appear in the formula for calculation of $\theta$ with the Equitest system (although these forces are not small in conditions 3 to 6). This omission is due to the assumption that the heel and 
ankle are a single point in the Equitest system. We do not ignore these forces in our two-link model.

2. $\theta$ is calculated in the Equitest system with the moving average of the positions of the COPs, whereas we do not make such an assumption in our two-link model.

We note that the Equitest system does calculate the sway angle $(\theta)$ internally at each of the 2,000 points that are used to find the ES. But this value of $\theta$ is not reported numerically in the output data (it only plots the curve for $\theta$ versus time). Also, the system does not report the torque as an output.

With the use of Equations (1) to (4) and the data from this same subject for all trials, the Composite PSI and Composite ES scores (reported by the NeuroCom device) based on a two-link model are 59.4 and 51.0, respectively. Note that the Composite PSI was evaluated in the same manner as the Composite ES, i.e., the weighted average of PSI scores from six conditions of the SOT of a subject, with conditions 3, 4, 5, and 6 having three times the weight of conditions 1 and 2. Note that ES is similar regardless of whether an individual spends most of the time at the boundary (limit of stability) or in the middle region, although an individual has more chances of falling in the former case. However, PSI is likely to be different for these two cases, because it is based on the sway angle throughout the test duration. As a second example, for condition 5 we used data from two subjects having the same ES of 74. One subject spent $9.44 \mathrm{~s}$ at the boundary and the other subject spent $11.25 \mathrm{~s}$ at the boundary. The PSI calculated for the former subject is 61.37, whereas the PSI for the latter subject is 45.41 , which, as expected, is lower than that of the former who spent less time at the boundary. The sway angle trace is shown over time in Figure 3(a) and (b) for a 20 s trial for each of the two sample subjects. These data show that one can quantify a difference in stability using the Composite PSI for two subjects with the same Composite ES. Because one of the two subjects spends more time near the limit of stability boundary, the PSI is indicating an important balance-related difference between these two sample subjects that is not apparent with the use of the NeuroCom-based ES. We determined the boundary region as any part of the sway path where the subject's sway was greater than the average on either the anterior or posterior side.

Data from 30 subjects (10 civilians with chronic fatigue syndrome [CFS], 10 veterans with medically unexplained symptoms, and 10 healthy controls) were (a)

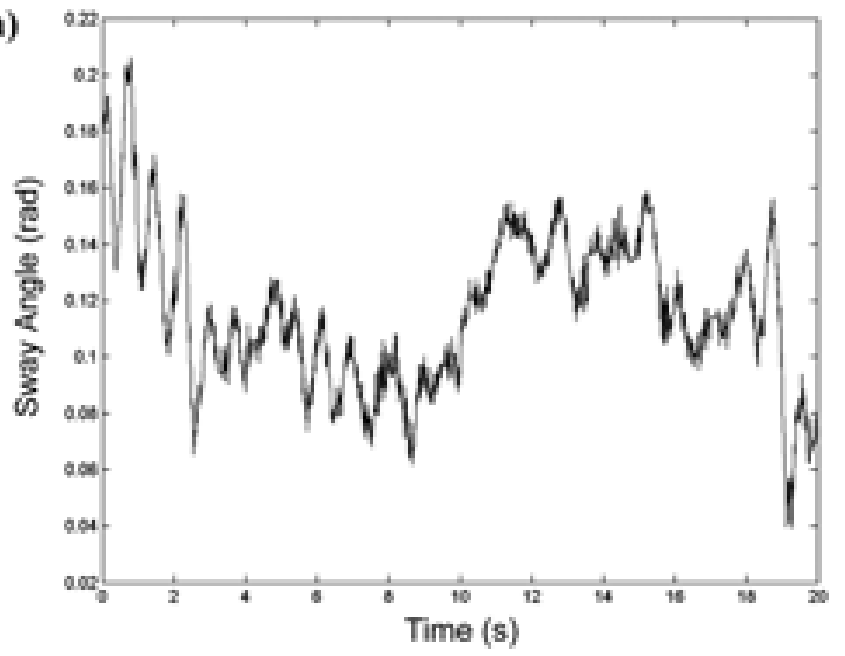

(b)

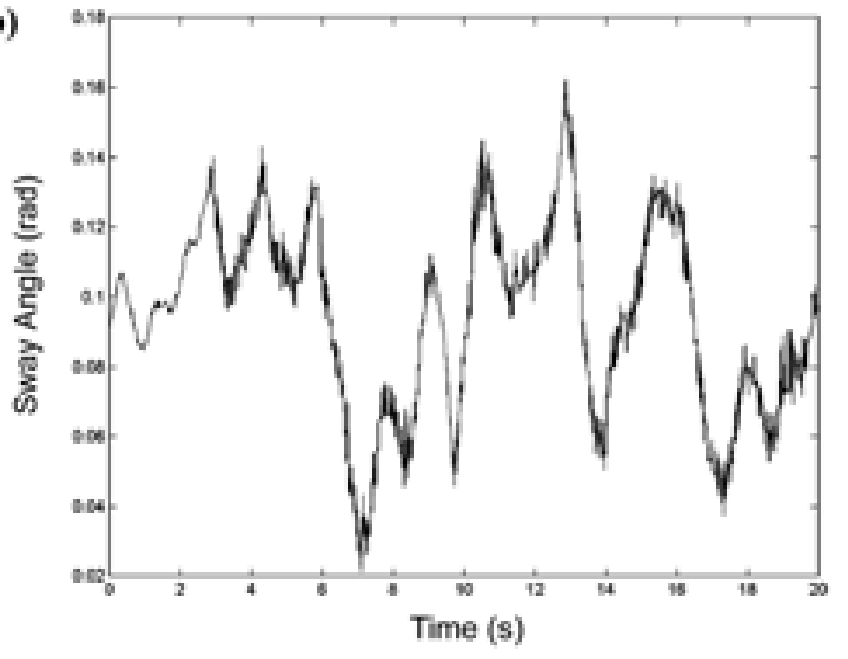

Figure 3.

Sway angle trace over time: (a) Subject 1-time spent near boundary $=9.44 \mathrm{~s}$, equilibrium score $=74$, Postural Stability Index $=61.37$. (b) Subject 2 - time spent near boundary $=11.25 \mathrm{~s}$, equilibrium score $=74$, Postural Stability Index $=45.41$.

also compared with the Composite ES score computed by the NeuroCom EquiTest System and the Composite PSI developed here (Table). These subjects gave informed consent and the protocols were approved by the East Orange Department of Veterans Affairs Medical Center Institutional Review Board (IRB) and the University of Medicine and Dentistry of New Jersey-Newark IRB. All subjects performed all trials in each condition of the SOT of the Equitest. We calculated Composite PSI in the same way as in the first example. The diagnostic groups of CFS (based on a strict case definition [12]) and veterans with unexplained symptoms were chosen for this analysis because they have balance problems. These two groups 
JRRD, Volume 41, Number 5, 2004

Table.

Experimental details of all 30 subjects.

\begin{tabular}{|c|c|c|c|c|}
\hline Patient & Age & $\begin{array}{c}\text { Composite } \\
\text { ES }\end{array}$ & $\begin{array}{c}\text { Composite } \\
\text { PSI }\end{array}$ & $\begin{array}{c}\text { Average } \\
\text { Sway Angle } \\
\text { (rad) }\end{array}$ \\
\hline \multicolumn{5}{|l|}{ Veteran } \\
\hline 1 & 34 & 66 & 59.402 & 0.098 \\
\hline 2 & 36 & 81 & 60.726 & 0.083 \\
\hline 3 & 55 & 60 & 67.763 & 0.048 \\
\hline 4 & 78 & 52 & 45.209 & 0.116 \\
\hline 5 & 37 & 84 & 56.218 & 0.14 \\
\hline 6 & 34 & 55 & 59.601 & 0.123 \\
\hline 7 & 55 & 66 & 55.735 & 0.106 \\
\hline 8 & 51 & 56 & 51.314 & 0.086 \\
\hline 9 & 56 & 71 & 63.868 & 0.046 \\
\hline 10 & 37 & 53 & 63.943 & 0.081 \\
\hline \multicolumn{5}{|l|}{ Healthy } \\
\hline 11 & 49 & 75 & 65.373 & 0.07 \\
\hline 12 & 30 & 62 & 61.214 & 0.091 \\
\hline 13 & 28 & 79 & 65.513 & 0.105 \\
\hline 14 & 55 & 77 & 57.654 & 0.126 \\
\hline 15 & 27 & 64 & 74.765 & 0.045 \\
\hline 16 & 54 & 61 & 50.08 & 0.114 \\
\hline 17 & 52 & 78 & 57.271 & 0.119 \\
\hline 18 & 22 & 80 & 58.599 & 0.083 \\
\hline 19 & 23 & 71 & 66.796 & 0.054 \\
\hline 20 & 28 & 90 & 56.047 & 0.08 \\
\hline \multicolumn{5}{|l|}{ CFS } \\
\hline 21 & 48 & 69 & 68.107 & 0.034 \\
\hline 22 & 23 & 79 & 60.544 & 0.127 \\
\hline 23 & 55 & 65 & 57.018 & 0.064 \\
\hline 24 & 54 & 68 & 80.996 & 0.031 \\
\hline 25 & 48 & 74 & 59.691 & 0.078 \\
\hline 26 & 33 & 78 & 57.867 & 0.1 \\
\hline 27 & 52 & 65 & 53.667 & 0.107 \\
\hline 28 & 48 & 62 & 61.876 & 0.084 \\
\hline 29 & 32 & 75 & 67.774 & 0.106 \\
\hline 30 & 48 & 48 & 52.667 & 0.046 \\
\hline
\end{tabular}

come to our center for clinical and research studies. The control group was used as a comparison. Figures 4 (all 30 subjects combined) and $\mathbf{5}$ (separate for controls, CFS, and veterans) show the relationship between Composite ES and average sway angle. Average sway angle is an important facet of balance [13-15], and thus should be related strongly to a measure of postural stability. These figures show that the relationship between Composite ES and average sway angle is small $\left(R^{2}=0.07\right)$ and opposite the

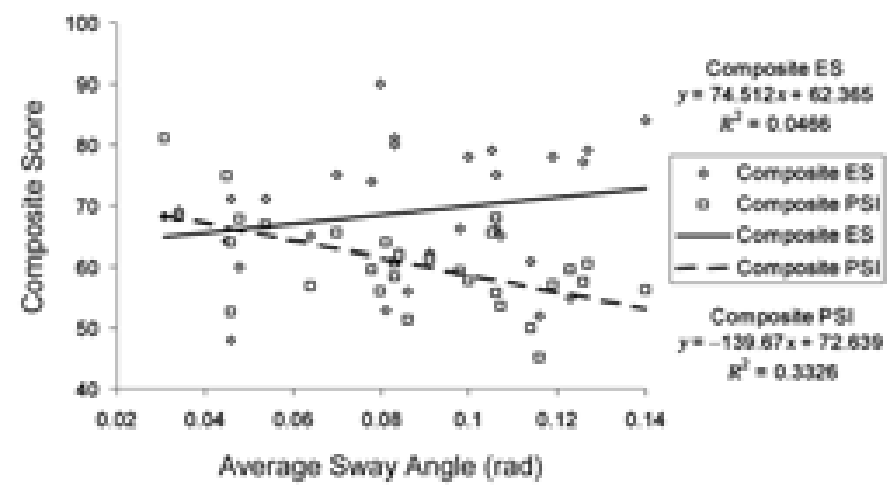

Figure 4.

Relationship between Composite Postural Stability Index (squares) and average angle of sway, and between Composite equilibrium score (diamonds) and average angle of sway of all subjects.

expected direction (i.e., as average sway angle gets larger, the Composite ES is better). Conversely, as the average sway angle increases, the Composite PSI decreases, as would be expected, and this relationship is strong $\left(R^{2}=\right.$ 0.50). These data document that Composite PSI provides more useful information about balance, since it is more strongly related to an important aspect of stability, namely average sway angle, than is Composite ES. These two measures tell us something different about a subject's performance and are not directly comparable. Composite ES gives us information about a subject's overall stability, compared with the normative total sway of $12.5^{\circ}$. PSI, on the other hand, reflects sway over the entire time history of a person's sway, not just their maximal sway. PSI is the percentage ratio of total stabilizing torque, and total destabilizing torque with a ratio of 100 indicating perfect stability. Thus, the PSI does not rely on any normative assumptions and reflects the entire sway history.

\section{DISCUSSION}

We have developed a formula for a PSI that includes the mass and height of the subject as well as the ankle torque explicitly. These parameters are absent in the formula for the NeuroCom EquiTest-derived ES. The ES reflects the total anterior-posterior sway, which likely depends on the cumulative effect of a subject's mass, height, and ankle torque. However, the ES assumes an angle of $12.5^{\circ}$ as the limit of stability for all individuals, irrespective of mass, height, age, or sex and is insensitive to different combinations of anterior-posterior sway. 
(a)

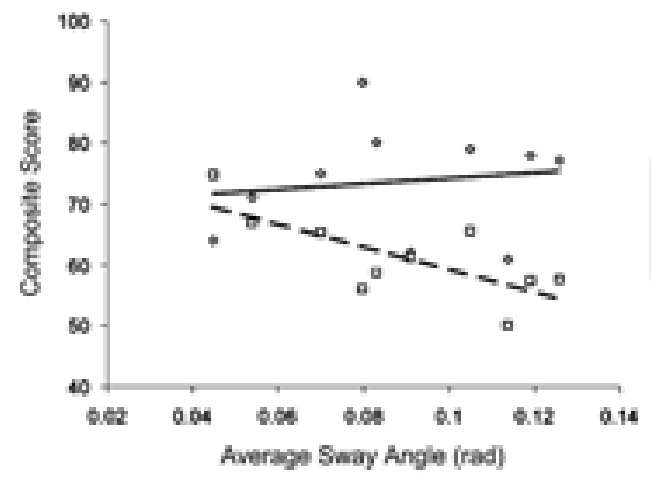

(c)

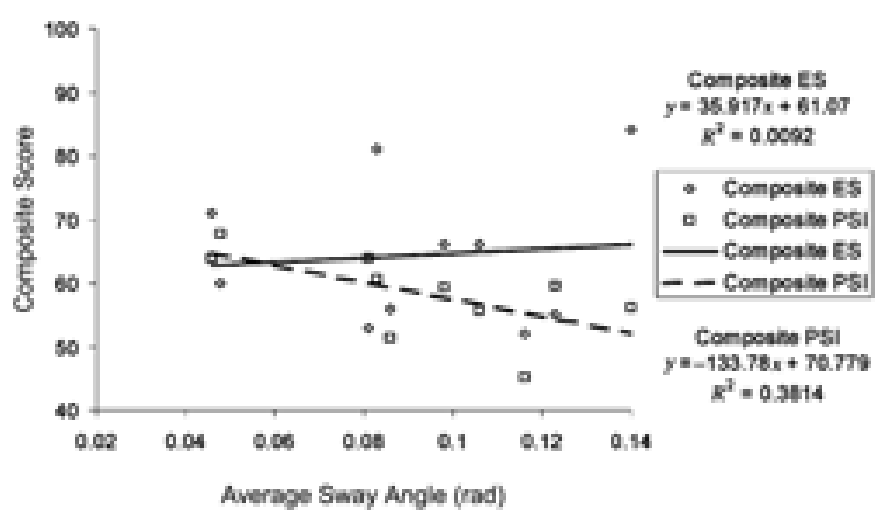

(b)
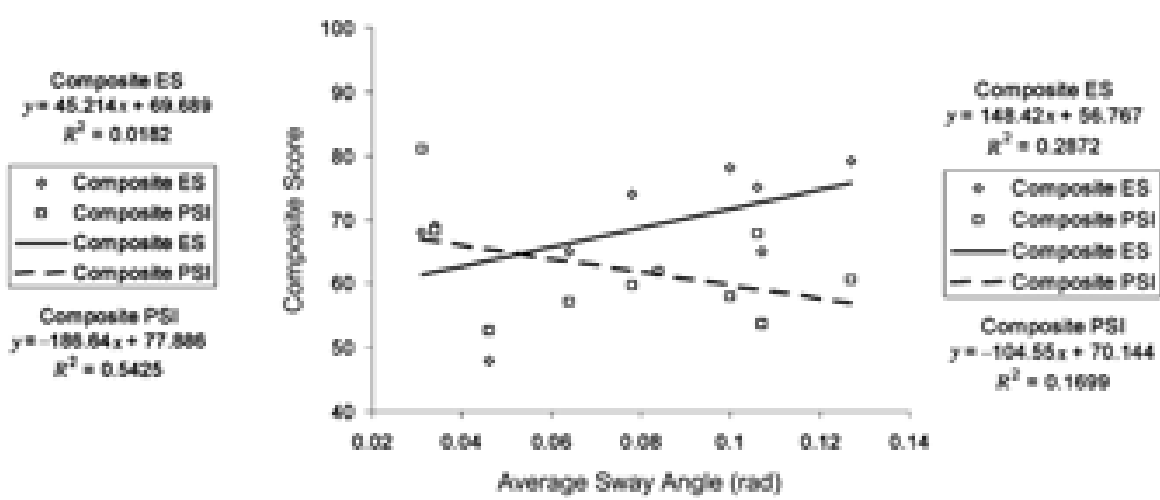

Figure 5.

Relationship between Composite Postural Stability Index (PSI) (squares) and average sway angle (rad), and between Composite equilibrium score (ES) (diamonds) and average angle of sway for (a) healthy control subjects, those subjects with (b) chronic fatigue syndrome, and (c) veterans with unexplained symptoms.

Moreover, the ES only considers the two extreme values of the sway angle in a given trial, not the sway angle at each data point. Thus, only two readings out of 2,000 measurements are considered for a $20 \mathrm{~s}$ trial, whereas the PSI computation includes the sway angle at every data point for each trial. Thus, the PSI uses data derived from multiple time points for an individual and considers a greater array of biomechanical variables that affect postural stability during quiet standing than does the ES. Note that the main purpose of this paper is not to quantitatively compare Composite ES with Composite PSI in the same link model, since they convey different meanings. Rather, our purpose is to propose a PSI that is conceptually very different from the ES and not directly comparable.

Software modification of the NeuroCom Equitest computer to provide a calculation of PSI should be a straightforward exercise. Once the software is incorpo- rated, the computer should be able to print PSI in addition to ES, since the PSI computation takes a fraction of a second of computer time. The SOT itself requires no modification. Our formula for PSI also can allow us to study the variability of oscillations of the COM in assessing stability [16], which will be the subject of future research.

\section{CONCLUSIONS}

We propose here a new measure of postural stability, the PSI, which provides a biomechanically based measure of postural stability that uses both vertical and horizontal force data from each sampled time point rather than simply the minimum and maximum sway values. Individual differences in postural stability thus are considered without appeal to normative assumptions about sway limits. We propose that the PSI provides a more 
JRRD, Volume 41, Number 5, 2004

biomechanically sound measure of postural stability that may have good clinical utility.

\section{ACKNOWLEDGMENTS}

We would like to thank Christina Rumage, Chrissy Giubilo, and Caixia Zhao for assisting in the data collection.

\section{REFERENCES}

1. Prieto TE, Myklebust JB, Myklebust BM. Characterization and modeling of postural steadiness in the elderly: A review. IEEE Trans Rehabil Eng. 1993;1:26-34.

2. Fabio RB, Emasthi A, Paul S. Validity of visual stabilization used with computerized dynamic platform posturography. Acta Otolaryngolica (Stockholm). 1998;118:449-54.

3. Johansson R, Magnusson M, Fransson PA, Karlberg M. Multi-stimulus multi-response posturography. Mathemat Biosci. 2001;174:41-59.

4. Berg KO, Wood-Dauphinee SL, Williams JI, Maki B. Measuring balance in the elderly: validation of an instrument. Can J Public Health. 1992;83 Suppl 2:S7-S11.

5. Horak FB. Clinical assessment of balance disorders. Gait Posture. 1997;6:74-86.

6. SmartEquitest System operator's manual. Clackamas (OR): NeuroCom International; 2001.

7. Balance Master operator's manual. Clackamas (OR): NeuroCom International; 2001.

8. Popovic MR, Pappas IPI, Nakazawa K, Keller T, Morari M, Dietz V. Stability criterion for controlling standing in able-bodied subjects. J Biomechan. 2000;33:1359-68.
9. Alexander NB, Shepard N, Gu MJ, Schultz A. Postural control in young and elderly adults when stance is perturbed: Kinematics. J Gerontol Med Sci. 1992;47:M79-M87.

10. Shepard N, Schultz A, Alexander NB, Gu MJ, Boismier T. Postural control in young and elderly adults when stance is challenged: Clinical versus laboratory measurements. Ann Otol Rhinol Laryngol. 1993;102:508-17.

11. Ji Z, Findley T, Chaudhry H, Bukiet B. Computational method to evaluate ankle muscle stiffness with ground reaction forces. J Rehabil Res Dev. 2004;41(2):207-14.

12. Fukuda K, Straus SE, Hickie I, Sharpe MC, Dobbins JG, Komaroff $\mathrm{A}$. The chronic fatigue syndrome: a comprehensive approach to its definition and study. International Chronic Fatigue Syndrome Study Group. Ann Int Med. 1994;121:953-59.

13. Lee J, Fujimoto N, Batiner A, Cervo F, Meyer J, Rubin C, McLeod K. Prediction of fall risk in the elderly: Time dependent measures of postural sway dynamics. Meeting of Orthopaedic Research Society, San Francisco, CA; 2001.

14. Stalenhoef PA, Diederiks JP, Knottnerus JA, Kester AD, Crebolder HF. A risk model for the prediction of recurrent falls in community-dwelling elderly: A prospective cohort study. J Clin Epidemiol. 2002;55:1088-94.

15. Stel VS, Smit JH, Pluijm SM, Lips P. Balance and mobility performance as treatable risk factors for recurrent falling in older persons. J Clin Epidemiol. 2003;56:659-68.

16. van Emmerick RE, van Wegen EE. On the functional aspects of variability in postural control. Exerc Sport Sci Rev. 2002;30:177-83.

Submitted for publication September 16, 2003. Accepted in revised form April 9, 2004. 